\title{
Identificación de Rickettsia andeanae en dos regiones de Chile
}

Katia Abarca, Javier López, Gerardo Acosta-Jamett y Constanza Martínez-Valdebenito

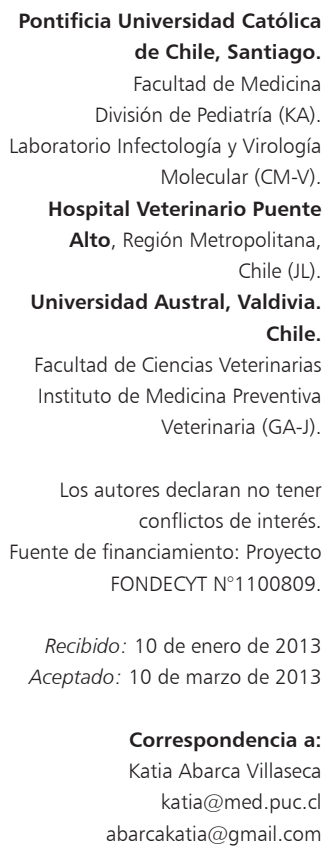

Pontificia Universidad Católica de Chile, Santiago.

Facultad de Medicina División de Pediatría (KA) Laboratorio Infectología y Virología Molecular (CM-V)

Hospital Veterinario Puente Alto, Región Metropolitana, Chile (JL) Universidad Austral, Valdivia. Chile.

Facultad de Ciencias Veterinarias Instituto de Medicina Preventiva Veterinaria (GA-J).

Los autores declaran no tener conflictos de interés. Fuente de financiamiento: Proyecto

FONDECYT N¹100809.

Recibido: 10 de enero de 2013 Aceptado: 10 de marzo de 2013

Correspondencia a: Katia Abarca Villaseca katia@med.puc.c abarcakatia@gmail.com

\section{Introducción}

$\mathrm{L}$ as rickettsias constituyen agentes emergentes de creciente reconocimiento en el mundo, debido en gran parte a la mayor disponibilidad de técnicas moleculares para su identificación ${ }^{1}$. Son transmitidas a hospederos vertebrados por vectores artrópodos como garrapatas, pulgas, piojos y ácaros, siendo las garrapatas, tanto de animales silvestres como domésticos, los vectores más frecuentemente implicados ${ }^{2}$. En Chile se han identificado tres especies de garrapatas caninas: Rhipicephalus sanguineus o garrapata café del perro y Amblyomma tigrinum en zonas rurales de la zona centro sur del país ${ }^{3-5} \mathrm{y}$ recientemente, $A$. triste en un sector rural cercano a Arica ${ }^{6}$. En el país se han identificado dos especies de Rickettsia en artrópodos: Rickettsia felis, encontrada en pulgas de gatos y en $R$. sanguineus ${ }^{7-9}$ y Candidatus 'Rickettsia andeanae', en un espécimen de $A$. triste de Arica ${ }^{6}$.

Rickettsia andeanae pertenece al grupo etiológico de las fiebres manchadas ${ }^{10} \mathrm{y}$ fue reportada por primera vez en un espécimen de Amblyomma maculatum y uno de Ixodes boliviensis del Norte del Perú en $2004^{11} \mathrm{y}$ posteriormente en $A$. maculatum en E.U.A. ${ }^{12-16}$ y en $A$. parvum, A. pseudoconcolor y A. tigrinum en Argentina ${ }^{17,18}$. Más recientemente se la ha identificado en $A$. maculatum recolectados de personas en E.U.A. ${ }^{19}$. A la fecha no se han confirmado casos de infección por $R$. andeanae en humanos ni en animales, por lo que se desconoce su rol patógeno. Su nomenclatura actual lleva el prefijo "Candidatus", utilizado para especies nuevas, aún no completamente caracterizadas. Recientemente ha sido cultivada en tres líneas celulares, a partir de un espécimen de A. maculatum recolectado en Virginia, E.U.A. ${ }^{20}$.

Los objetivos del estudio fueron: determinar la presencia de agentes rickettsiales en garrapatas extraídas de perros domésticos de dos regiones de Chile; describir la distribución urbano/rural de los agentes identificados; y comparar filogenéticamente las especies encontradas con otras previamente publicadas.

\section{Material y Métodos}

Se realizó un estudio transversal, descriptivo, en las regiones de Coquimbo y La Araucanía, donde se efectuó un muestreo domiciliario, aleatorio, en las ciudades de Coquimbo y Angol (La Araucanía) y un muestreo por conveniencia en localidades rurales cercanas a ambas ciudades. El tamaño de muestra calculado para otro objetivo (seroprevalencia de agentes ehrlichiales) fue de 97 perros en cada localidad. Un equipo constituido por un veterinario y un auxiliar de veterinaria visitó las casas 
seleccionadas entre octubre de 2011 y febrero de 2012. Cada vivienda fue geo-referenciada, registrándose las coordenadas UTM. En viviendas con mascotas caninas y cuyos dueños accedieron a participar, se procedió a la toma de consentimiento informado por un adulto responsable y se ingresó al estudio a un perro, el que fue examinado por el veterinario extrayéndose manualmente garrapatas cuando estaban presentes. Las garrapatas extraídas fueron depositadas en frascos con alcohol de $70^{\circ}$ y transportadas al Laboratorio de Infectología de la Pontificia Universidad Católica de Chile en Santiago a temperatura ambiente, para su procesamiento.

La determinación de especies se realizó examinando los especímenes con microscopio estereoscópico tri-ocular con zoom entre 6,7 y 45x, marca Olympus, utilizando las claves taxonómicas publicadas ${ }^{21-23}$.

Posteriormente fueron maceradas para la extracción de ADN genómico utilizando el 'Wizard Genomic DNA Purification Kit'TM (Promega, Madison, WI, USA). El ADN extraído fue sometido a amplificación por RPC del gen de la citrato sintetasa ( $g l t \mathrm{~A})$, gen común para las rickettsias del grupo de las fiebres manchadas y que permite clasificarlas en este grupo, utilizado los partidores CS-78 y CS-323 de acuerdo a condiciones previamente descritas ${ }^{24}$. Los productos de RPC de $401 \mathrm{pb}$ fueron visualizados en un gel de agarosa al 2\% teñido con Syb®safe (Invitrogen $^{\circledR}$ ), cortados, purificados usando el kit QIAquick PCR Purification Kit ${ }^{\mathrm{TM}} \mathrm{y}$ enviados a secuenciar (Macrogen, Bethesda, Maryland, USA).

De las muestras positivas para gltA se amplificó el gen ompA, utilizado para la identificación de la especie de Rickettsia, por ser un gen menos conservado. Se utilizaron los partidores 190.70F-A1 y 190.602R-A2 de acuerdo a condiciones previamente descritas ${ }^{19}$. Los productos de RPC de $512 \mathrm{pb}$ fueron visualizados en un gel de agarosa al 2\% teñido con Syb®safe (Invitrogen ${ }^{\circledR}$ ), cortados, purificados y secuenciados con la metodología previamente indicada.

Las secuencias de gltA y ompA fueron editadas manualmente usando el programa Biedit v7.1.3.0 para la generación de una secuencia consenso por cada muestra analizada. La secuencias consenso fueron analizadas mediante la herramienta Blastn.

Las secuencias de ompA de mayor tamaño obtenido se utilizaron para efectuar alineamiento con otras secuencias y construir una tabla de identidad así como el árbol filogenético.

El alineamiento se realizó utilizando la herramienta Clustalw construyendo una tabla de identidad, dividida en dos secciones, la inferior izquierda con la identidad entre las secuencias de gltA (gen con poca divergencia entre las distintas especies) y la superior derecha con las identidades de ompA (gen con mayor variabilidad en las secuencias de distintas especies).
Para la reconstrucción filogenética se utilizó el método de máxima verosimilitud, basado en el modelo de Jones y cols., que toma en cuenta el tipo de sustitución aminoacídica en secuencias del tipo codificantes ${ }^{20}$. El árbol consenso fue inferido de 500 replicados (boostrap) ${ }^{21}$.

Con las coordenadas UTM y utilizando el programa Arc Gis 10 se construyeron mapas de las dos regiones estudiadas indicando la presencia de garrapatas con y sin material genético de Rickettsia.

El estudio fue aprobado por los Comités de Ética de la Dirección de Investigación y de Bienestar Animal de la Facultad de Medicina de la Pontificia Universidad Católica de Chile y por el Comité de Ética de CONICYT. Las autoridades de salud de ambas regiones fueron informadas de la ejecución del estudio.

\section{Resulltados}

\section{Población en estudio}

Se visitaron 942 viviendas: 583 urbanas y 359 rurales. El porcentaje de viviendas con mascota canina en la Región de Coquimbo fue 58\% en las viviendas urbanas y $92 \%$ en las rurales y en la Región de La Araucanía, $57 \%$ en las viviendas urbanas y $98 \%$ en las rurales. Se enrolaron en el estudio 462 viviendas (entre 112 y 124 por localidad), con mascota canina y cuyos dueños aceptaron participar; en cada vivienda se enroló un perro (Tabla 1).

\section{Especies de garrapatas}

De los 462 perros examinados, entre 38 y $66 \%$ estaban parasitados por garrapatas en las distintas localidades (Tabla 1). El análisis de especie de garrapata fue realizado en $86 \%$ de los perros, correspondiendo a $R$. sanguineus (garrapata café del perro) en $92 \%$ de los casos. Se encontró solamente esta especie en las ciudades de Coquimbo y Angol. En las localidades rurales se encontró además garrapatas del género A. tigrinum, en 27 y $36 \%$ de los perros de las regiones de Coquimbo y La Araucanía, respectivamente. Diez perros presentaban ambas especies de garrapatas en forma simultánea (Tabla 1).

Los especímenes de $A$. tigrinum presentaron un cuerpo oval alargado con un escudo ornamentado característico, mucho más pequeño en la hembra y careciendo de tubérculos en la superficie ventral de los festones. Los machos presentaron su característica espina larga y aguda en la Coxa I y IV y una espina corta y obtusa en la Coxa II y III; las hembras difieren sólo en la espina de la Coxa IV que es de igual tamaño que las otras ${ }^{21-23}$ (Figura 1).

\section{Identificación molecular de Rickettsias}

En los especímenes de $A$. tigrinum se obtuvo amplificación del fragmento del gen gltA en 24 de 31 analizados, en tanto, de un total de 225 especímenes de $R$. sanguineus 


\begin{tabular}{|c|c|c|c|c|c|}
\hline Localidad & $\begin{array}{c}\text { Perros evaluados } \\
\text { n }\end{array}$ & $\begin{array}{l}\text { Perros con garrapatas } \\
\qquad n \quad(\%)\end{array}$ & $\begin{array}{l}\text { Perros con garrapatas evaluadas } \\
\text { n } \quad(\%)\end{array}$ & $\begin{array}{l}\text { Rhipicephalus sanguineus } \\
\text { n (\%) }\end{array}$ & $\begin{array}{l}\text { Amblyomma tigrinum } \\
\text { n (\%) }\end{array}$ \\
\hline Coquimbo urbano & 114 & $60 \quad(53)$ & $59(98)$ & $59 \quad(100)$ & 0 \\
\hline Coquimbo rural & 112 & $70 \quad(63)$ & $66 \quad(94)$ & $55 \quad(83)^{*}$ & $18(27)^{*}$ \\
\hline Angol urbano & 124 & $82 \quad(66)$ & $66 \quad(81)$ & $66(100)$ & 0 \\
\hline Angol rural & 112 & $43 \quad(38)$ & $28 \quad(65)$ & $(75)^{\star *}$ & $10(36)^{* *}$ \\
\hline Total & 462 & $255 \quad(55)$ & 219 (86) & $201(92)^{\star \star *}$ & $28(13)^{* \star *}$ \\
\hline
\end{tabular}
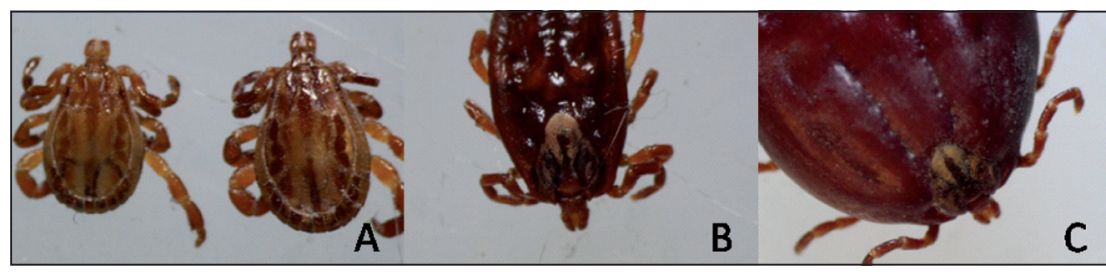

Figura 1. Especímenes de Amblyomma tigrinum. Machos (A), hembra (B) y hembra ingurgitada (C).

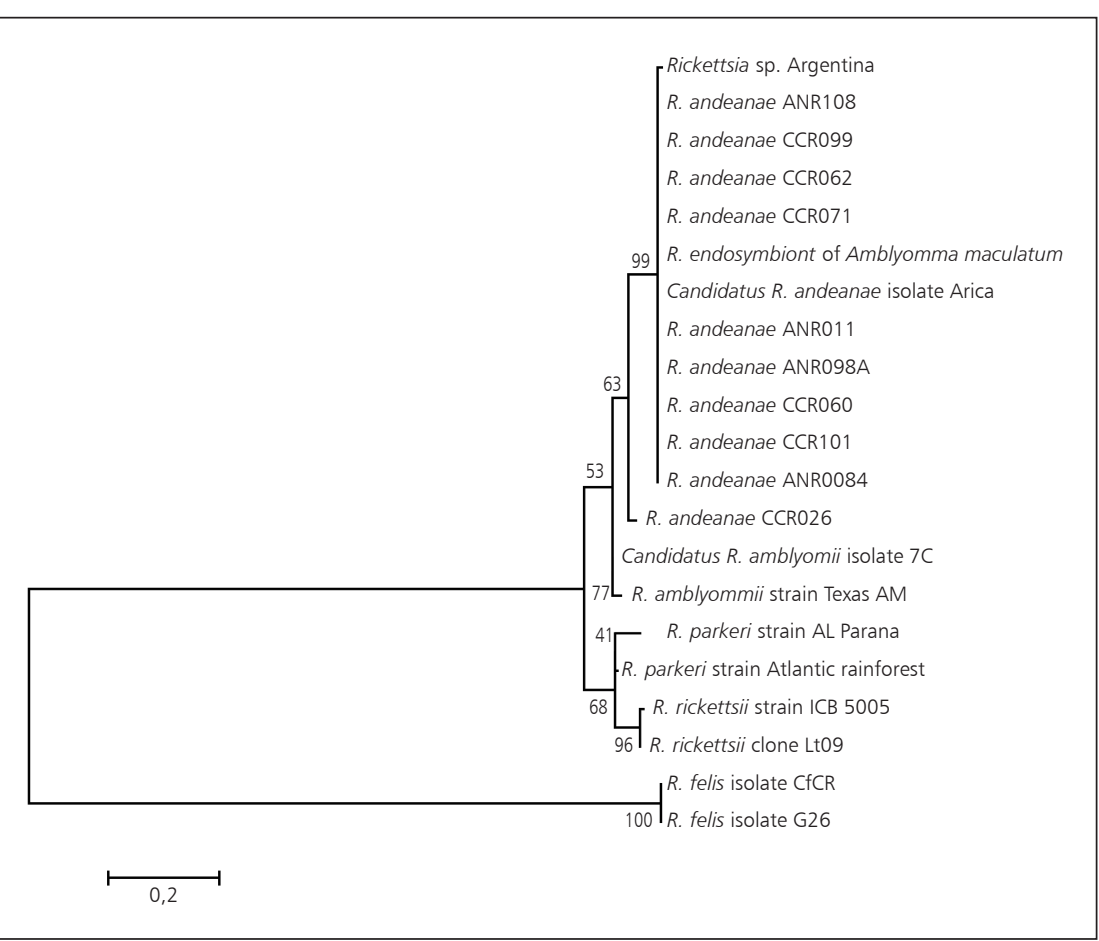

Figura 2. Análisis filogenético usando 10 de las 12 secuencias obtenidas de la amplificación del gen ompA, además de 11 secuencias obtenidas del GenBank. (JN393870.1, EF372578.1, JF694093.1, JN990593.1, JN126318.1, JQ906784.1, JN384014.1, EU826512.1, JN180850.1, EF194096.1, JQ690645.1). El análisis de las secuencias se realizó mediante el método de máxima verosimilitud basado en el modelo Jones et $a^{25}$. El árbol consenso fue inferido de 1.000 replicados (boostrap) y es representativo de la historia evolutiva de las 21 secuencias analizadas. $C R=$ Coquimbo rural; ANR $=$ Angol rural. analizados, sólo uno resultó positivo para este gen. De las 25 muestras positivas para gltA, 21 fueron secuenciadas. La secuencia obtenida de $R$. sanguineus fue menor a 150 $\mathrm{pb}$, lo que sugiere una rickettsia del grupo etiológico de fiebres manchadas, sin ser de tamaño adecuado para la comparación con otras secuencias. De las restantes 20, todas provenientes de $A$. tigrinum, se obtuvo secuencias de longitud adecuada, las que presentaron entre 98,4 y $100 \%$ de identidad entre ellas; el análisis en Blastn de las secuencias consenso mostró $99 \%$ de identidad con Candidatus 'R. andeanae' encontrada en A. triste de Arica (JN180850.1) ${ }^{6}$ y con Rickettsia spp. (EF451004.1) reportada en $A$. parvum de Argentina ${ }^{17}$.

De estas 20 muestras se logró amplificar el gen ompA en 16, obteniéndose información de sus secuencias en 12 de ellas, todas provenientes de A. tigrinum; la muestra correspondiente a $R$. sanguineus fue negativa para este gen, por lo que no pudo ser identificada la especie y no fue incorporada al análisis. Las 12 secuencias de ompA mostraron corresponder a Candidatus 'R. andeanae', con $99 \%$ de identidad. Estas corresponden a garrapatas extraídas de ocho perros de las zonas rurales de Coquimbo y cuatro de Angol.

Dos de estas secuencias resultaron con menos de 200 $\mathrm{pb}$ por lo que no fueron utilizadas para la matriz de identidad ni para la reconstrucción filogenética, usándose los restantes 10 fragmentos que tenían una longitud de $421 \mathrm{pb}$. Nueve de estas secuencias son homólogas entre sí (100\% identidad) y se agruparon juntas en una de las ramas del árbol, en conjunto con Candidatus 'R. andeanae' aislado desde A. triste en Arica (JN180850.1) ${ }^{6}$ y con Rickettsia spp. aislada en Argentina (EU826512) ${ }^{14}$, con un soporte de boostrap de $98 \%$. La otra secuencia se ubica en otra rama muy cercana, teniendo un ancestro común a las nueve anteriores, correspondiendo también a Candidatus 'R. andeanae' (Figura 2).

En la Tabla 2 se muestran las identidades entre dos secuencias tipo provenientes de este estudio, una de cada localidad rural, y otras ocho obtenidas desde GenBank. 
Tabla 2. Matriz de identidad entre dos secuencias obtenidas en este estudio y ocho previamente publicadas

\begin{tabular}{|c|c|c|c|c|c|c|c|c|c|c|}
\hline Secuencias & R. amblyommii & R. felis & R. parkeri & R. rickettsii & R. rickettsii & $\begin{array}{l}\text { Rickettsia sp } \\
\text { 'Argentina' }\end{array}$ & $\begin{array}{l}\text { R. andeanae } \\
\text { ANR108 }\end{array}$ & $\begin{array}{l}\text { R. andeanae } \\
\text { CCR060 }\end{array}$ & $\begin{array}{l}\text { R. amblyommii } \\
\text { Texas A\&M }\end{array}$ & $\begin{array}{c}R \text { andeanae } \\
\text { Arica }\end{array}$ \\
\hline R. amblyommii & ID & 0,45 & 0,93 & 0,90 & 0,91 & 0,94 & 0,95 & 0,95 & 0,99 & 0,95 \\
\hline R. felis & 0,93 & ID & 0,45 & 0,44 & 0,44 & 0,46 & 0,46 & 0,46 & 0,45 & 0,46 \\
\hline R. parkeri & 0,99 & 0,92 & ID & 0,96 & 0,96 & 0,90 & 0,90 & 0,90 & 0,92 & 0,90 \\
\hline R. rickettsii & 0,98 & 0,92 & & ID & 0,99 & 0,87 & 0,87 & 0,87 & 0,90 & 0,87 \\
\hline R. rickettsii & 0,98 & 0,92 & 0,98 & 0,98 & ID & 0,87 & 0,88 & 0,88 & 0,91 & 0,88 \\
\hline $\begin{array}{l}\text { Rickettsia sp. } \\
\text { 'Argentina' }\end{array}$ & 0,99 & 0,92 & 0,99 & 0,98 & 0,98 & ID & 0,99 & 0,99 & 0,94 & 0,99 \\
\hline $\begin{array}{l}\text { R. andeanae } \\
\text { ANR108 }\end{array}$ & 0,99 & 0,92 & 0,98 & 0,98 & 0,98 & 0,99 & ID & 0,99 & 0,94 & 1 \\
\hline $\begin{array}{l}\text { R. andeanae } \\
\text { CCR060 }\end{array}$ & 0,99 & 0,92 & 0,99 & 0,98 & 0,98 & 1 & 0,99 & ID & 0,94 & 0,99 \\
\hline R. amblyommii & 0,99 & 0,92 & 0,99 & 0,98 & 0,98 & 1 & 0,98 & 1 & ID & 0,94 \\
\hline $\begin{array}{l}\text { R. andeanae } \\
\text { Arica }\end{array}$ & 0,99 & 0,92 & 0,99 & 0,98 & 0,98 & 1 & 0,99 & 1 & 1 & ID \\
\hline
\end{tabular}

Las secuencias de ompA se muestran a la derecha y arriba (destacado en gris) y las de gltA abajo a la izquierda. Se muestran en negrita las mayores similitudes de las dos secuencias tipo obtenidas en este estudio (ANR108 y CCR060).

La mayor identidad se encontró con Candidatus 'R. andeanae' descrita en Arica ${ }^{6}$.

\section{Distribución geográfica de $R$. andeanae}

La Figura 3 muestra la localización geográfica de las viviendas de las zonas rurales de las regiones de Coquimbo y La Araucanía en cuyos perros se encontró garrapatas $A$. tigrinum infectadas y no infectadas con $R$. andeanae. Dado que todas las garrapatas de las ciudades de Coquimbo y Angol correspondieron exclusivamente a $R$. sanguineus y que todas ellas resultaron negativas para $R$. andeanae, no se incluyeron en los mapas.

\section{Discusión}

El presente estudio documenta la presencia de Candidatus 'R. andeanae' en dos distantes regiones de Chile, lo que se suma a su reciente hallazgo en el extremo norte del país, en una localidad rural cercana a la ciudad de Arica ${ }^{6}$. Estos hallazgos indican que esta especie, de reciente descripción, se encuentra ampliamente distribuida en el país. Su presencia en Chile se limitaría a garrapatas del género Amblyomma (A. triste en la Región de Arica y Parinacota y $A$. tigrinum en las regiones de Coquimbo y La Araucanía) y a las zonas rurales, lo que es concordante con lo documentado por otros investigadores y con el hábitat silvestre de esta garrapata ${ }^{26}$.

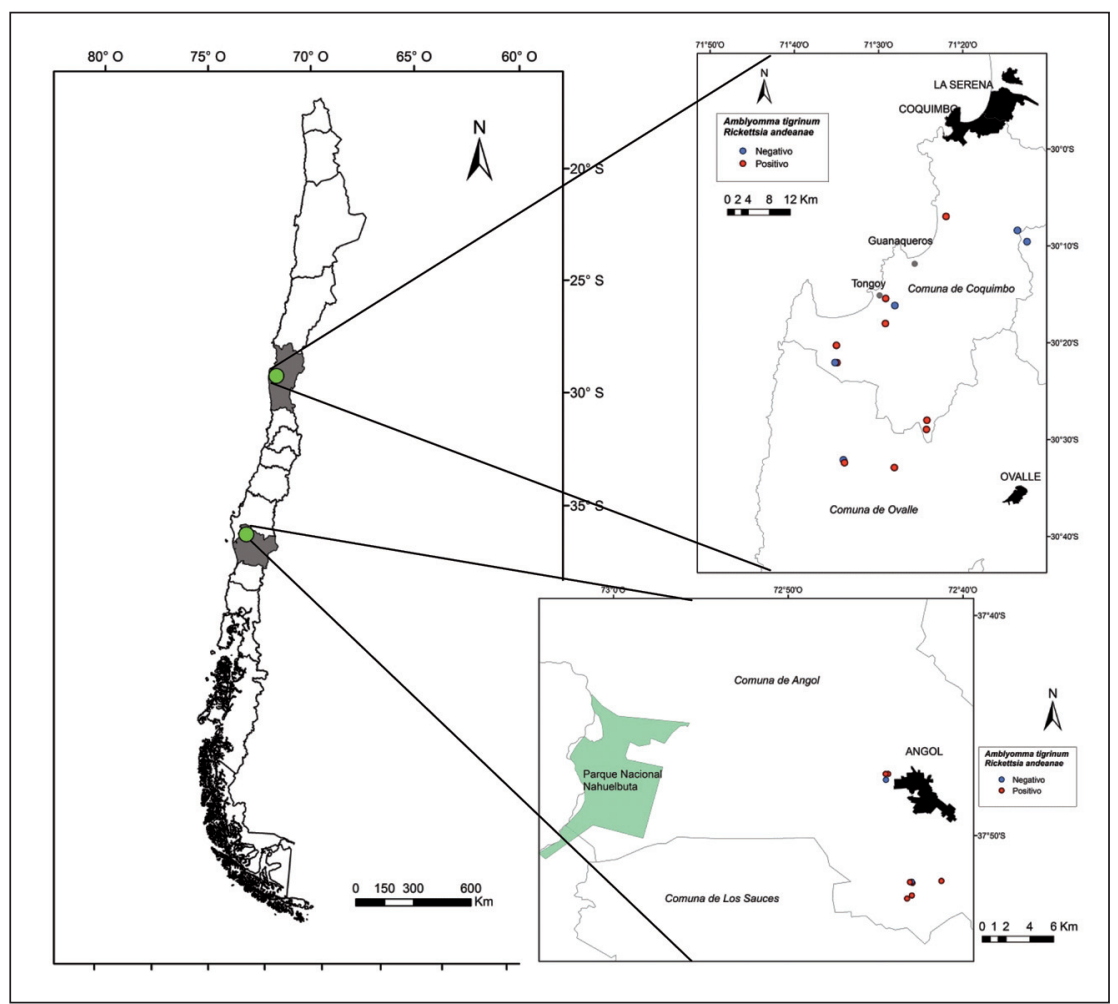

Figura 3. Área de estudio en las regiones de Coquimbo y La Araucanía indicándose las garrapatas de A. tigrinum que resultaron positivas y negativas a Candidatus 'Rickettsia andeanae'. 
En Chile, A. tigrinum había sido descrita en las regiones de Valparaíso y Bío-Bío ${ }^{3,28}$. Nuestros hallazgos indican que su distribución es más extensa en el país. Esto, sumado a que su presencia ha sido reportada en toda Sudamérica $^{21}$, refuerza el concepto que esta especie se encuentra presente en áreas de diversa latitud y clima del continente americano. Investigadores argentinos destacan la plasticidad de esta especie de garrapata, puesto que se la encuentra en climas tan diversos como los Andes Patagónicos, la región amazónica, El Chaco y la pampa argentina, lo que contrasta con la especificidad de clima de otras especies de Amblyomma $^{27}$. Su ciclo de vida requiere de hábitats rurales o silvestres, puesto que los estadios inmaduros (larvas y ninfas) se encuentran en aves ${ }^{29,30}$ y roedores silvestres ${ }^{31,32}$, lo que explica su presencia exclusiva en localidades rurales. Los adultos parasitan cánidos domésticos y silvestres y ocasionalmente al hombre; se los encuentra todo el año, pero son más numerosos en verano $^{27}$. Se ha especulado que los estadios inmaduros ocupan refugios o escudos naturales que permiten mitigar condiciones climáticas extremas, explicando su presencia en áreas de gran contraste ecológico ${ }^{27}$.

Estudios moleculares indican que $R$. andeanae se ubica en el grupo etiológico de las fiebres manchadas ${ }^{10}$; su primer hallazgo, en Perú, se hizo en dos garrapatas recolectadas de un área previamente afectada por un brote de cuadros febriles y dos muertes ${ }^{10,11}$. El estudio inicial de este brote mostró evidencia serológica de Leptospira, Coxiella burnetti y Rickettsia sp y evidencia molecular de la presencia de una rickettsia del grupo etiológico de las fiebres manchadas en algunos pacientes febriles, sin que se pudiera precisar la especie ${ }^{33}$. A la fecha aún no se ha determinado el rol patógeno de $R$. andeanae para animales o el hombre, pero los antecedentes mencionados sugieren que puede haber sido un agente etiológico en el mencionado brote de Perú. Es importante considerar que varias especies de Rickettsia inicialmente se consideraban apatógenas y posteriormente se ha documentado su virulencia, como es el caso de Rickettsia parkeri cuya patogenicidad se conoció 65 años después de su descripción inicial ${ }^{34}$. Por ello, algunos expertos consideran que toda nueva especie de Rickettsia encontrada en un hospedero invertebrado debe considerarse como potencialmente patógena para los humanos ${ }^{35}$. Su reciente propagación exitosa en cultivos celulares permitirá avanzar tanto en su validación como una nueva especie de Rickettsia como en determinar su rol patógeno ${ }^{20}$.

Candidatus Rickettsia andeanae corresponde a la primera rickettsia identificada en garrapatas $A$. tigrinum en nuestro país y como se ha mencionado, había sido recientemente encontrada en garrapatas $A$. triste en Arica ${ }^{6}$. Otras dos especies de Rickettsia han sido identificadas en

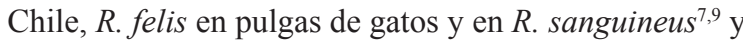
Orientia tsutusugamushi, identificada en un paciente con antecedentes de mordedura por sanguijuelas ${ }^{36}$. A nuestro conocimiento, este último es el único caso humano de rickettsiosis documentado en el país en la época actual. La inespecificidad de los síntomas clínicos, el desconocimiento de los agentes y vectores presentes en las distintas zonas geográficas y la ausencia de técnicas de diagnóstico explican el importante sub-diagnóstico característico de muchas infecciones rickettsiales ${ }^{1,37}$.

Destaca la elevada tasa de infección por $R$. andeanae encontrada en las garrapatas A. tigrinum, ya que se pudo confirmar su presencia en 12 de 31 especímenes analizados (39\%). Estudios previos con más de 10 especímenes estudiados han mostrado cifras variables de infección por este agente en distintos artrópodos: 1-5\% en $A$. maculatum de E.U.A. ${ }^{12,13}, 4 \%$ en $A$. tigrinum, $54 \%$ en $A$. pseudoconcolor y $68 \%$ y $94 \%$ en $A$. parvum de Argentina ${ }^{17,18}$. La prevalencia de infección por Rickettsia en los vectores es un elemento que puede ayudar a estimar el riesgo de transmisión de estos agentes en diferentes áreas geográficas.

Otra implicancia de la descripción de $A$. tigrinum en varias regiones del país, así como de $A$. triste en Arica, radica en que estas especies son vectores de $R$. parkeri, una rickettsia de reconocido poder zoonótico que ha sido identificada en varios países del continente, tanto en vectores como causando infecciones en personas ${ }^{34,38-43}$. Recientemente se ha reportado co-infección por $R$. parkeri y $R$. andeanae en $A$. maculatum de la costa del Misisipi, en E.U.A. ${ }^{13}$.

El conocimiento de la distribución de los vectores así como de la asociación vector-agente infeccioso ejemplificado en este caso (garrapatas de la especie Amblyomma-R. andeanae) puede permitir deducir áreas de distribución de determinados agentes infecciosos y por ende, de riesgo para la salud humana y/o animal. De comprobarse un rol patogénico de $R$. andeanae para los humanos, los antecedentes aportados en este estudio permitirán predecir zonas de riesgo para infecciones del tipo fiebres manchadas, alertar a los médicos clínicos a sospechar e identificar tales casos y a las autoridades a planificar efectivas medidas de control vectorial.

Agradecimientos. A Gonzalo Vieyra, Fabiola Núñez, Paola Oyarzún, Victory Angel y Victoria Neehdman quienes realizaron el muestreo domiciliario. A Paulina Lepe que realizó la identificación taxonómica de las garrapatas. A Marcelo Labruna, por su experta revisión de la identificación taxonómica y sus siempre valiosas sugerencias y comentarios.

\section{Resumen}

Introducción: Candidatus 'Rickettsia andeanae', especie de reciente reconocimiento y rol patógeno no precisado, 
ha sido identificada en garrapatas del género Amblyomma en Perú, E.U.A. y Argentina y recientemente en Chile, en un espécimen de Amblyomma triste de un sector rural de Arica. Objetivo: Determinar presencia de agentes rickettsiales en garrapatas de perros domésticos de dos regiones de Chile. Métodos: Estudio transversal, descriptivo, en las regiones de Coquimbo y La Araucanía, con muestreo domiciliario en las ciudades de Coquimbo y Angol y localidades rurales cercanas, en primavera-verano 2011-2012. Se examinó un perro por vivienda, recolectando garrapatas si estaban presentes; luego de su identificación taxonómica fueron sometidas a amplificación y secuenciación de los genes gltA y ompA. Resultados: Se examinaron 462 perros, 255 tenían garrapatas (55\%). En las ciudades se encontró exclusivamente Rhipicephalus sanguineus; en las localidades rurales además Amblyomma tigrinum. Las secuencias obtenidas en 12 especímenes de A. tigrinum correspondieron a Candidatus 'Rickettsia andeanae'. Conclusiones: Se documenta presencia de $R$. andeanae en dos regiones distantes de Chile, lo que se suma a su reciente hallazgo en el extremo norte del país. Este nuevo agente rickettsial se limita a garrapatas del género Amblyomma y a zonas rurales, lo que concuerda con lo documentado en otros países y con el hábitat de esta garrapata.

\section{Referencias bibliográficas}

1.- Parola P, Paddock C D, Raoult D. Tick-borne rickettsioses around the world: emerging diseases challenging old concepts. Clin Microbiol Rev 2005; 18: 719-56.

2.- Parola P, Raoult D. Ticks and tickborne bacterial diseases in humans: An emerging infectious threat. Clin Infect Dis 2001; 32: 897-928.

3.- Tagle L, Álvarez V. Rectificación de diagnóstico: Amblyomma tigrinum Koch en lugar de Amblyomma maculatum Koch. Bol Chil Parasitol 1959; 14 (3): 56-7.

4.- González-Acuña D, Guglielmone A A. Ticks (Acari: Ixodoidea: Argasidae, Ixodidae) of Chile. Exp Appl Acarol 2005; 35: 147-63.

5.- Abarca K, López J, Acosta-Jamett G, Lepe P, Labruna M. Especies de garrapatas en caninos de localidades urbanas y rurales de Arica y de la Región Metropolitana. CO-16, XXVIII Congreso Chileno de Infectología 2011. Rev Chilena Infectol 2011; 28 (Supl 2): S117.

6.- Abarca K, López J, Acosta-Jamett G, Lepe P, Soares J F, Labruna M B. A third amblyomma species and the first tick-borne Rickettsia in Chile. J Med Entomol 2012; 49: 219-22.

7.- Labruna M B, Ogrzewalska M, Moraes-Filho J, Lepe P, Gallegos J L, López J. Rickettsia felis in Chile. Emerg Infect Dis 2007; 13: 1974-5.

8.- Abarca K, López J, Acosta-Jamet G, Martínez-Valdebenito C. Rickettsia felis in Rhipicephalus sanguineous from two distant Chilean cities. Vector-borne Zoonotic Dis 2013; 13, in press.

9.- Abarca K, López J, Acosta Jamett G, Martínez C, Lepe P, Labruna M. Dos especies de Rickettsias identificadas en pulgas y garrapatas de caninos de zonas urbanas y rurales de Arica y de la Región Metropolitana, Chile. P-37, XXVIII Congreso Chileno de Infectología 2011. Rev Chilena Infectol 2011; 28 (Supl 2): S79.

10.- Jiang J, Blair PJ, Vidal F, Moron C, Céspedes M, Anaya E, et al. Phylogenetic analysis of a novel molecular isolate of spotted fever group rickettsiae from northern Peru. Ann N Y Acad Sci 2005; 1063: 337-42.

11.- Blair P J, Jiang J, Schoeler G B, Moron C, Anaya E, Céspedes M, et al. Characterization of spotted fever group Rickettsiae in flea and tick specimens from Northern Peru. J Clin Microbiol 2004; 42: 4961-7.

12.- Paddock C D, Fournier P E, Summer J W, Goddard J, Elshenawy Y, Metcalfe M G, et al. Isolation of Rickettsia parkeri and identification of a novel spotted fever group Rickettsia sp. from Gulf Coast ticks (Amblyomma maculatum) in the United States. Appl Environ Microbiol 2010; 76: 2689-96.

13.- Ferrari F A G, Goddard J, Paddock C D. Varela-Stokes A S. Rickettsia parkeri and Candidatus Rickettsia andeanae in Gulf Coast ticks, Mississippi, USA. Emerg Infect Dis 2012; 18: 1705-7.

14.- Wright C L, Nadolny R M, Jiang J, Richards A L, Sonenshine D E, Gaff H D, et al. Rickettsia parkeri in gulf coast tick, southeastern Virginia, USA. Emerg Infect Dis 2011; 17: 896-8.

15.- Fornadel C M, Zhang X, Smith J D, Paddock C D, Arias J R, Norris D E. High Rates of Rickettsia parkeri infection in Gulf Coast ticks (Amblyomma maculatum) and Identification of Candidatus 'Rickettsia andeanae' from Fairfax County, Virginia. Vector-Borne Zoonotic Dis 2011; 11 (12): 1535-9.

16.- Varela-Stokes A S, Paddock C D, Engber B, Toliver M. Rickettsia parkeri in Amblyomma maculatum ticks, North Carolina, USA, 2009-2010. Emerg Infect Dis 2011; 17 (12): 2350-3.

17.- Pacheco R C, Moraes-Filho J, Nava S, Brandão P E, Richtzenhain L J, Labruna M B. Detection of a novel spotted fever group rickettsia in Amblyomma parvum ticks (Acari: Ixodidae) from Argentina. Exp Appl Acarol 2007; 43: 6-71.

18.- Tomassone L, Núñez P, Ceballos L A,
Furtler R E, Kitron U, Farber M. Detection of "Candidatus Rickettsia sp. strain Argentina" and Rickettsia bellii in Amblyomma ticks from Northern Argentina. Exp Appl Acarol 2010; 52: 93-100.

19.- Jiang J, Stromdahl E Y, Richards A L. Detection of Rickettsia parkeri and Candidatus 'Rickettsia andeanae' in Amblyomma maculatum Gulf Coast ticks from humans in the United States. Vector-Borne Zoonotic Dis 2012; 12 (3): 175-82.

20.- Luce-Fedrow A, Wright C H, Gaff H D, Sonenshine D E, Hynes W L, Richards A L. In vitro propagation of Candidatus 'Rickettsia andeanae' isolated from Amblyomma maculatum. FEMS Immunol Med Microbiol 2012; 64: 74-81.

21.- Jones E K, Clifford C M, Keirnas J E, Khols G M. Ticks of Venezuela (Acarina: Ixodoidea) with a key to the species of Amblyomma in the Western Hemisphere, Brigham Young Univ Sci Bull 1972.

22.- Estrada Peña A, Guglielmone A A, Mangold A J, Castellá J. A description of Amblyomma tigrinum Koch; A. naumani Ribaga and $A$. testudinis (Conil) immatures (sic) (Acarina: Ixodidae). Folia Parasitol 1993; 40: 147-53.

23.- Santa Cruz A C M, Sarmiento N F, Comolli J A, Gonzáles J A, González A O. Descripción de los principales aspectos morfológicos de Amblyomma tigrinum Koch, 1844 (Arthropoda: Ixodidae) de Hydrochaeris hydrochaeris por microscopio de luz y electrónico de barrido. Comunicaciones Científicas y Tecnológicas Universidad del Nordeste 2006. Resumen V-050.

24.- Labruna M B, Whitworth T, Horta M C, Bouyer D H, McBride J W, Pinter A, et al. Rickettsia species infecting Amblyomma cooperi ticks from an area in the State of São Paulo, Brazil, where Brazilian spotted fever is endemic. J Clin Microbiol 2004; 42: 90-8.

25.- Jones D T, Taylor W R, Thornton J M. The rapid generation of mutation data matrices from 
protein sequences. Comput Appl Biosci 1992; 8: 275-82.

26.- Felsenstein J. Confidence limits on phylogenies: An approach using the bootstrap. Evolution 1985; 39: 783-91.

27.- Guglielmone A A, Mangold A J, Hadami A. Amblyomma tigrinum Koch, 1844 (Acari: Ixodidae) en relación con la fitogeografía del centro-norte argentino, hospedadores y disribución estacional. Estación Experimental Agropecuaria INTA. Rafaela. Documento anuario 2000, pág 121.

28.- Muñoz L, Casanueva M. Garrapatas (Acari: Ixodida) en perros de la ciudad de Concepción, Chile. Arch Med Vet 2002; 34 (1): 131-4.

29.- Venzal J M, Castro O, Cabrera P, de Souza C; Claramunt S. Garrapatas de aves de Uruguay: antecedentes y nuevas contribuciones. IX Congreso Brasileiro de Ornitología; Curitiba; Paraná, Brasil 2001; pág 86.

30.- González Acuña D, Venzal J, Skewes O, Rubilar Contreras L, Daugschies A, Guglielmone A A. Primer registro de los estadios inmaduros de Amblyomma tigrinum (Acari: Ixodidae) en pájaros silvestres en Chile. Acarology 2004; 33: 153-6.

31.- Nava S, Mangold A J, Guglielmone A A. The natural host of larvae and nymphs of Amblyomma tigrinum Koch, 1844 (Acari: Ixodidae). Vet Parasitol 2006; 140: 124-32.
32.- Labruna M, Souza S L P, Menezes A C, Horta M C, Pinter A, Gennari S M. Life-cycle and host specifity of Amblyomma tigrinum (Acari: Ixididae) under laboratory conditions. Experimental Applied Acarol 2002; 24: 113-25.

33.- Blair P J, Scholere G B, Moron C, Anaya E, Caceda R, Céspedes M, et al. Evidence of rickettsial and leptospira infections in Andean northern Peru. Am J Trop Med Hyg 2004; 70 : 357-363.

34.- Paddock C D, Summer K W, Comer J A, Zaki S R, Goldsmith C S, Goddard J, et al. Rickettsia parkeri: a newly recognized cause of spotted fever rickettsiosis in the United States. Clin Infect Dis 2004; 38: 805-11.

35.- Labruna M B, Mattar S, Nava S, Bermúdez S, Venzal J M, Dolz G, et al. Rickettsioses in Latin America, Caribbean, Spain and Portugal. Rev MVZ Córdoba 2011; 16 (2): 2435-57.

36.- Balcells M E, Rabaglaiti R, García P, Poggi H, Oddó D, Concha M, et al. Endemic scrub thypus-like illness, Chile. Emerg Infect Dis 2011; 17 (9): 1659-63.

37.- Zavala J, Ruiz A, Zavala J. Spotted fever Rickettsiae. Their immunodominant proteins and immune response. Rev Med Chile 2004; 132: 381-7.

38.- Whitman T J, Richards A L, Paddock C D, Tamminga C L, Sniezek P J, Jiang J, et al. Rickettsia parkeri infection after tick bite,
Virginia. Emerg Infect Dis 2007; 13: 334-6.

39.- Sumner J W, Durden L A, Goddard J, Stromdahl E Y, Clark K L, Reeves W K, et al. Gulf coast ticks (Amblyomma maculatum) and Rickettsia parkeri, United States. Emerg Infect Dis 2007; 13 (5):751-2.

40.- Conte V, Parrilla G, de Meneghi D, Tomassone L. Prevalence of Rickettsia infection in dogs and detection of Rickettsia parkeri in Amblyoma tigrinum ticks in Cochabamba Department, Bolivia. Book of Proceedings, VI International Conference on Ticks and Tick-Borne Pathogens. Buenos Aires, Argentina 2008; pág. 076.

41.- Venzal J M, Estrada-Peña A, Castro O, de Souza CG, Félix ML, Nava S, et al. Amblyomma triste Koch 1844 (Acari: Ixodidae): hosts and seasonality of the vector of Rickettsia parkeri in Uruguay. Vet Parasitol 2008; 155: 104-9.

42.- Nava S, Elshenawy Y, Eremeeva M E, Sumner J W, Mastropaolo M, Paddock CD. Rickettsia parkeri in Argentina. Emerg Infect Dis 2008; 14 (12): 1894-7.

43.- Paddock C D, Fournier P E, Summer J W, Goddard J, Elshenawy Y, Metcalfe M G, et al. Isolation of Rickettsia parkeri and identification of a novel spotted fever group Rickettsia $s$. from Gulf Coast ticks (Amblyomma maculatum) in the United States. Appl Environ Microbiol 2010; 76: 2689-96. 